\title{
Magnetic Oscillations at a Metal Surface
}

\author{
Predicted 40 years ago, magnetic Friedel oscillations are finally observed \\ in the layers beneath an iron surface.
}

\author{
By Michael Schirber
}

$\mathrm{n}$ a magnetic material, the magnetization at the surface

is not necessarily the same as in the bulk. At the surface, the reduced number of neighbors can, for example, increase or decrease the polarization of electron spins, producing higher or lower magnetization. Theorists predicted 40 years ago that the magnetization on iron surfaces should vary with depth in the form of so-called Friedel oscillations. Using specially grown iron films and gamma-ray spectroscopy, a team has confirmed those predictions [1]. The researchers believe that this method will become a powerful tool for studying spintronic devices.

Friedel oscillations are spatial modulations of electron spin or charge density that result from a defect or boundary in a material. Researchers have observed Friedel oscillations on thin-film surfaces, but the oscillations are also expected with depth for certain magnetic materials. For iron, theory predicts that the (001) crystal should exhibit layer-by-layer oscillations from the surface toward the bulk.

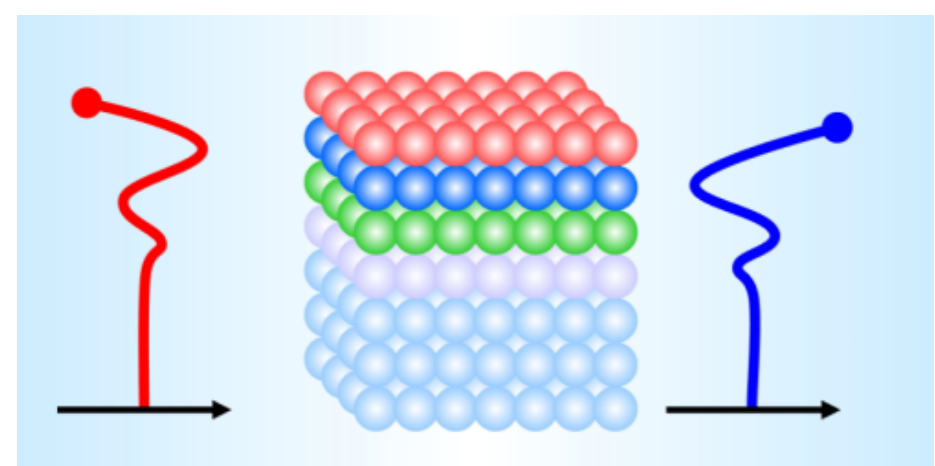

Credit: T. Mitsui/ National Institutes for Quantum and Radiological Science and Technology
To observe the oscillations, Takaya Mitsui, from the National Institutes for Quantum and Radiological Science and Technology in Japan, and colleagues first grew iron films with single layers of the isotope iron-57 implanted at various depths. Performing iron-57 Mössbauer spectroscopy with a brilliant synchrotron source, the researchers identified a spectral feature associated with an internal magnetic field called the hyperfine field. By theoretically deriving a relation between the hyperfine field and the magnetization, the team confirmed that the magnetization is highest at the topmost atomic layer and then oscillates in strength down to the fourth atomic layer. Such information could help in tuning the spin transport properties of thin materials.

Michael Schirber is a Corresponding Editor for Physics based in Lyon, France.

\section{REFERENCES}

1. T. Mitsui et al., "Magnetic Friedel oscillation at the Fe(001) surface: Direct observation by atomic-layer-resolved synchrotron radiation ${ }^{57}$ Fe Mössbauer spectroscopy," Phys. Rev. Lett. 125, 236806 (2020). 